\title{
Prevalence of Musculoskeletal Pain and Injuries in Gym Instructors
}

\author{
Nikita Shinde ${ }^{1}$, Priya Sahasrabuddhe ${ }^{2}$ \\ ${ }^{1}$ Clinical Physiotherapist (B.P.Th), Symbiosis University Hospital and Research Centre, Pune, India \\ ${ }^{2}$ Assistant Professor, Musculoskeletal Sciences, Smt. Kashibai Navale College of Physiotherapy, Pune, India \\ Corresponding Author: Nikita Shinde
}

\begin{abstract}
Background: The gym instructors perform high intensity work during their personal session or while assisting their clients during their workout session. This is one of the major reasons for various injuries and subsequent pain. This study evaluated the prevalence of musculoskeletal pain and injuries in gym instructors.

Objectives: To obtain the prevalence of musculoskeletal injuries and pain in gym instructors.

Methods: A cross sectional study was performed on 108 gym instructors from different fitness clubs. A self-administered questionnaire was used. Percentile analysis of the scores was done.

Results: $16 \%$ of gym instructors suffered with injures of shoulder, low back and knee. About $82 \%$ of gym instructors had pain in various body areas.

Conclusion: The prevalence of pain and injuries was high in gym instructors which demands fitness industries to adapt prevention strategies.
\end{abstract}

Keywords: Gym instructors, musculoskeletal pain, injury

\section{INTRODUCTION}

Musculoskeletal injuries and associated pain is a common consequence of work related hazards. Different causes of musculoskeletal pain are unusual or repetitive activities that put strain on the muscles, tendons and sprain the ligaments; it also can be the result of traumatic events due to jerky movements, falls, fracture or underlying musculoskeletal diseases. Musculoskeletal pain mostly affects the bone, muscle, ligaments and tendons. [1] There is an increase in the number of attendants in the fitness industry in the last $10-15$ years. The gym instructors in the industry helps an individual to increase the cardiovascular endurance, strengthen the muscle and bone, boosts immune system, improves mobility and balance, improves posture. Though there are numerous health related benefits of physical activity and exercise, a major disadvantage is the risk of injuries leading to pain. ${ }^{[2]}$ A descriptive epidemiological study proved that the prevalence of acute and overuse injuries was higher in high instruction loading as compared to low instruction loading and the common site was ankle and lower leg. The most frequent site of musculoskeletal pain was shoulder and neck. ${ }^{[2]}$

Fitness instructors have limited knowledge regarding the injuries and injury prevention. An epidemiological study has provided evidence regarding the overuse injuries seen in competitive cyclists and the common site is low back and knee. [3] Monotonous exercise loading was a predominant factor among the instructors. ${ }^{[4]}$ Cardio kickboxing has also become a popular activity and studies have found that, the most prevalent site of injuries in cardio kickboxing instructors are knee and hip. One of the wide spreading and upcoming program which consists of series of 
exercises is crossfit this consists of high intensity exercises which are performed rapidly with successive repetitions having no or less recovery time. The injury rate in crossfit is more as compared to Olympic weightlifting, distance running, track and field, rugby, or gymnastics. ${ }^{[6]}$

Common strength training exercise is squatting and to avoid overuse injury while performing it proper knowledge of loading and posture is important. In a previous study it was confirmed that, while performing restricted squats there was increase in the thoracic curvature leading to stresses at back, whereas performing unrestricted squats there was increase in knee ROM and at the trunk small amount of segmental movements were seen. [7] The aim of this study was to know the prevalence of musculoskeletal pain and injuries in gym instructors and the objectives were to obtain the period prevalence of musculoskeletal pain in gym instructors to obtain the prevalence of musculoskeletal injuries in gym instructors.

\section{METHODS}

Objectives: To obtain the prevalence of musculoskeletal injuries and pain in gym instructors.

Study Design: Cross-sectional observational study

Study Population: 108 gym instructors (90 males, 18 females) from fitness clubs of Pune were taken. Convenient sampling technique was done. Inclusion criteria was gym instructors who handled client manually, age group from 20 years old to 30 years old (young adult) population was taken, Minimum 3 years of work experience and minimum 6 hours of working per day. Gym instructors who worked part time, age below 20 years of age and zumba and yoga instructors were excluded from the study.

\section{Procedure}

According to inclusion criteria questionnaire was filled by gym instructors. All questions were compulsory to answer. Statistical and data analysis was done to analyze the results. A self-administered Questionnaire adapted from Orebro musculoskeletal pain questionnaire was used, face validity of the questionnaire was done. The questionnaire consists three components and eighteen questions. The three components include "site of pain or injury, frequency, intensity of the pain", "physical activities aggravating pain or injury", "ADLs can be performed in spite of pain or injury". The statement are rated from (score 0) completely agree to (score 10) completely disagree. A ten point scale is used. For measuring the intensity of pain NRS was used the validity and reliability of this scale is published.

\section{Statistical Analyses}

The data were analyzed using Microsoft Office Excel 2010. Mean and standard deviations were analyzed for physical activities aggravating pain or injury, ADLs can be performed in spite of pain or injury. Percentile analysis was done to analyze the results.

\section{RESULTS}

According to figure1 among 108 gym instructors pain was present in maximum number of male and female gym instructor as compared to injury. Pain is present more in male gym instructors as compared to female gym instructors.

According to Figure 3 maximum gym instructors including male and female had pain in shoulder(79\%), lowerback (18\%), knee (17\%), wrist $(11 \%)$, ankle (10\%), elbow (6\%), neck (4\%), hip (2\%), upper back $(1 \%)$, other $(1 \%)$. 

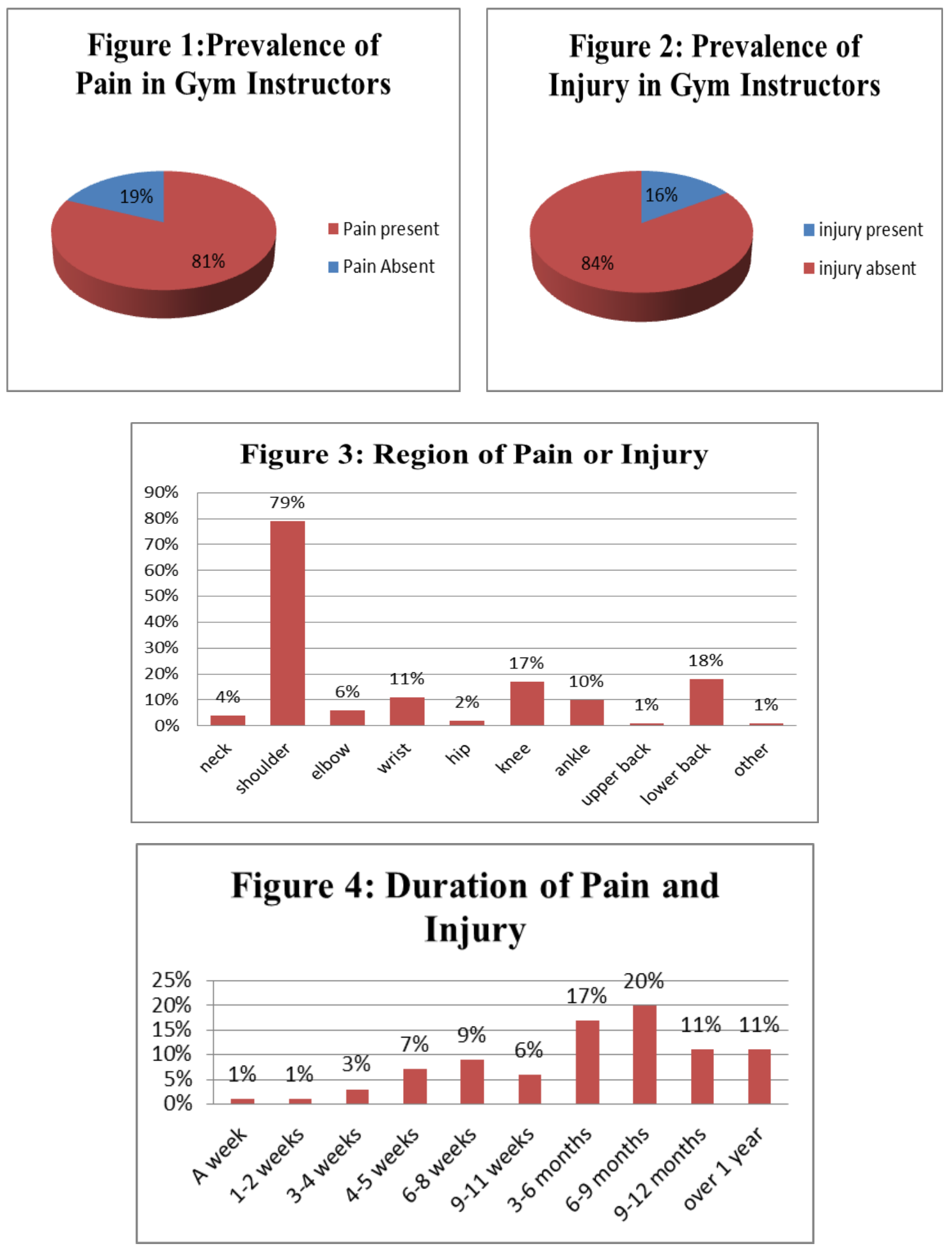

According to figure $420 \%$ of gym instructors suffered through pain or injury in 6-9 months, $17 \%$ of gym instructors suffered through pain or injury in 3-6 months, $11 \%$ of gym instructors suffered through pain or injury in 9-12 months, $11 \%$ of gym instructors suffered through pain or injury over 1 year, $9 \%$ of gym instructors suffered through pain or injury in 6-8 weeks, $7 \%$ of gym instructors suffered through pain or injury in 4-5 weeks, 3\% of gym instructors suffered through pain or injury in 1-2 weeks, $1 \%$ of gym instructors suffered through pain or injury in a week.

According to the figure 5 numbers of male gym instructors having mild pain were more compared to female gym instructors, numbers of male gym instructors having 
moderate pain were more compared to female gym instructors, numbers of male gym instructors having severe pain were more compared to female gym instructors.

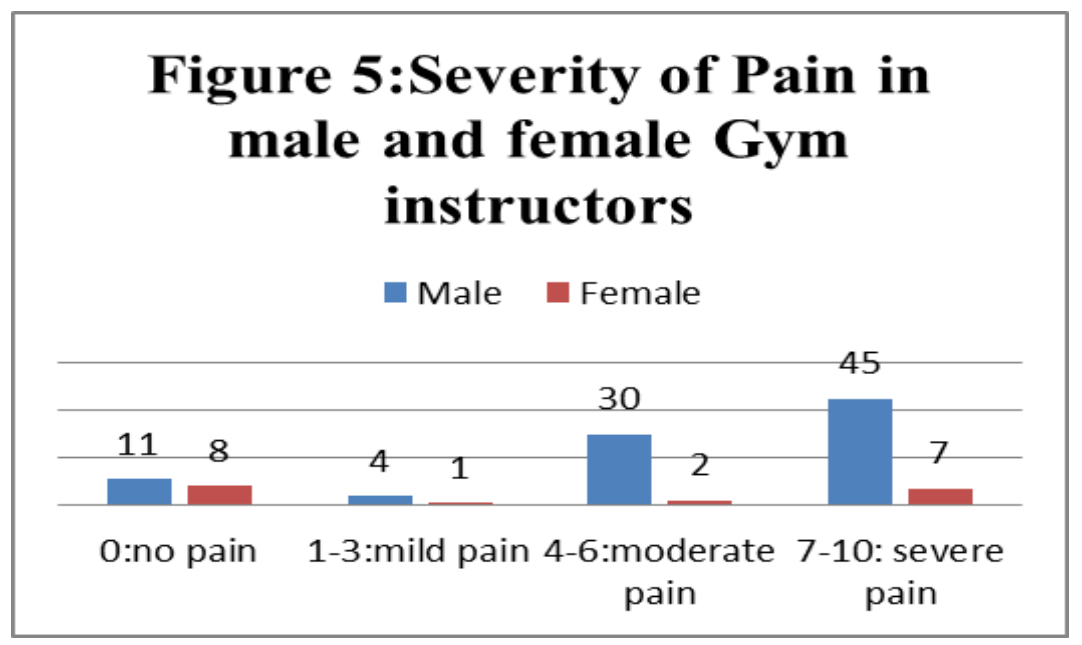

\section{DISCUSSION}

In the present study, $82 \%$ of gym instructors had pain and $16 \%$ of gym instructors had injury [figure 1]. The severity of pain in male gym instructors was more as compared to female gym instructors. One of the reasons can be maximum male gym instructors are undergoing high mechanical loading due to which there is an increase impact on the body weight, leading to strain on joints, ligaments, or tendons causing pain or injury. The injury rate while performing crossfit was high in male as compared to female. ${ }^{[8]}$

When we consider site of pain or injury, most affected regions are shoulder followed by low-back and knee [figure 3] and the reason can be shoulder joint provides mobility at the cost of stability. Shoulder joint is prone to instability as the muscles and ligaments holding the joint are stretched beyond limits. Stretching of shoulder ligaments causes pain that occurs either quickly or gradually. Frequent overhead activities such as abduction and flexion of arm produces pressure on the rotator cuff which makes the rotator cuff prone to impingement leading to pain or injury. A study done on Norwegian gym instructors confirmed that the most frequent site of pain during high instruction loading was shoulder and neck region. ${ }^{[2]}$ Whereas another study performed on cardio kickboxing instructors reported most affected regions were back, shoulder, elbow and wrist. The elbow and wrist region were noted to be the highest site of new injuries with no history of previous pain or injury. The most common type of injuries were sprain, strain and tendinitis. ${ }^{[5]}$ Reason for low back pain can be strain on the muscles or sprain at the ligaments. Strain of the muscles results due to heavy weight lifting during high intensity workout. Sudden twisting of spine while lifting weight will also cause injury to the lower back. In absence of core muscle training, lifting heavy weights can also be the reason of pain or injury. In a study they confirmed that in endurance sports repetitive action of flexion, extension and prolong hours of standing had less effects on LBP and a large mechanical loading performed from past year had large effects on LBP. ${ }^{[9]}$ The other most common region prone to injury or pain is knee joint and the possible reason can be spasm of thigh muscle, ligament sprains, tight hamstring and quadriceps. During heavy weight lifting there is excessive loading at the patellofemoral joint which may give rise to retropatellar pain. Heavy mechanical loading when performed with weights causes the ligaments to stretch leading to sprain of ligaments. Spasm of the muscles are caused due to lack of warm up and stretching activity. In one study they confirmed that, the most prevalent site of 
injuries in cardio kickboxing instructors were knee and hip. ${ }^{[5]}$

In the current study, maximum gym instructors $53(59 \%)$ had chronic type of pain and 36(40\%) gym instructors had acute type of pain [figure 4]. More often, acute micro injuries can lead to chronic injury which hamper the ability to perform work and affects the quality of life. Previous injury or trauma can also lead to chronic pain resulting in structural changes. Pain which lasts less than 3 months is (how to cite article) stated as acute pain ${ }^{[10]}$ and the pain that persist beyond the normal time expected for tissue healing (that is 3 months) is known as chronic pain. ${ }^{[11]}$

In the current study the severity of the pain is measured by NRS and maximum number of male gym instructors had more amount of moderate to severe type of pain as compared to female gym instructors [figure 5]. Many studies were conducted regarding the pain tolerance in gender, two of the studies confirm that women are at higher risk for developing pain disorders and women show high sensitivity towards noxious stimuli. ${ }^{[12,13]}$ However the number of female gym instructors were less than male gym instructors so ultimately the severity of pain was more in male gym instructor.

\section{CONCLUSION}

The prevalence of musculoskeletal pain in male gym instructors was $88 \%$ and in female gym instructors was $56 \%$ and the intensity of pain was moderate to severe in both genders. The prevalence of musculoskeletal injury in male was $18 \%$ and in female was $6 \%$. Most affected region was shoulder followed by low back and knee.

\section{Future scope of the study}

More data and area can be evaluated and type of injury can be studied in order to prevent or reduce the incidence of pain or injury.

\section{Acknowledgement: None}

\section{Conflict of Interest: None}

\section{Source of Funding: None}

\section{Ethical Approval: Approved}

\section{REFERENCES}

1. Paul Dieppe. Chronic Musculoskeletal Pain. Peninsula College of Medicine and Dentistry, Exeter, UK. BMJ 2013; 346:bmj.f3146

2. Solfrid Bratland-Sanda, Jorunn SundgotBorgen \& Grethe Myklebust (2015) Injuries and musculoskeletal pain among Norwegian group fitness instructors, European Journal of Sport Science, 15:8, 784-792

3. Clarsen, B., Krosshaug, T., \& Bahr, R. (2010). Overuse injuries in professional road cyclists. The American Journal of Sports Medicine, 38(12), 2494-2501

4. Garrick, J. G., Gillien, D. M., \& Whiteside, P. (1986). The epidemiology of aerobic dance injuries. The American Journal of Sports Medicine, 14(1), 67-72.

5. Romaine, L. J., Davis, S. E., Casebolt, K., \& Harrison, K. A.(2003). Incidence of injury in kickboxing participation. Journal of Strength and Conditioning Research, 17(3), 580-586.

6. Klimek C, Ashbeck C, Brook AJ, Durall C. Are Injuries More Common With CrossFit Training than Other Forms of Exercise? J Sport Rehabil. 2018 May 1; 27(3):295-299.

7. List R, Gülay T, Stoop M, Lorenzetti S. Kinematics of the trunk and the lower extremities during restricted and unrestricted squats. J Strength Cond Res. 2013 Jun; 27(6):1529-38.

8. Weisenthal BM, Beck CA, Maloney MD, DeHaven KE, Giordano BD. Injury Rate and Patterns Among CrossFit Athletes. Orthop J Sports Med. 2014 Apr 25;2(4):2325967114531177.

9. Foss IS, Holme I, Bahr R. The prevalence of low back pain among former elite crosscountry skiers, rowers, orienteerers, and nonathletes: a 10-year cohort study. Am J Sports Med. 2012 Nov;40(11):2610-6.

10. Susan Youssef. Clinical guidelines and evidence base for acute pain management The Pharmaceutical Journal, online DOI: 10.1211/PJ.2019.20206653

11. Shaquir Salduker, Eugene Allers, Sudha Bechan, R Eric Hodgson, Fanie Meyer, 
Helgard Meyer.et al., Practical approach to a patient with chronic pain of uncertain etiology in primary care: J Pain Res. 2019; 12: 2651-2662

12. Rollman GB, Lautenbacher S. Sex differences in musculoskeletal pain. Clin J Pain. 2001 Mar;17(1):20-4.
13. Fillingim RB. Sex, gender, and pain: women and men really are different. Curr Rev Pain. 2000;4(1):24-30.

How to cite this article: Shinde N, Sahasrabuddhe P. Prevalence of musculoskeletal pain and injuries in gym instructors. Int $J$ Health Sci Res. 2021; 11(4): 62-67. DOI: https://doi.org/10.52403/ijhsr.20210408 